\title{
Anti-angiogenic properties of artemisinin derivatives (Review)
}

\author{
TIANSHU WEI ${ }^{1,2}$ and $\mathrm{JU}_{\mathrm{LIU}}^{2}$ \\ ${ }^{1}$ School of Population and Global Health, The University of Melbourne, Victoria 3010, Australia; \\ ${ }^{2}$ Laboratory of Microvascular Medicine, Medical Research Center, Shandong Provincial Qianfoshan Hospital, \\ Shandong University, Jinan, Shandong 250014, P.R. China
}

Received June 12, 2016; Accepted July 27, 2017

DOI: $10.3892 /$ ijmm.2017.3085

\begin{abstract}
Angiogenesis, the process involving the development of new blood vessels from existing capillaries, is critical for growth and wound healing. However, pathological angiogenesis contributes to the pathogeneses of numerous diseases, including cancer, rheumatoid arthritis, diabetic retinopathy and macular degeneration. Hence, the inhibition of angiogenesis is an effective therapeutic approach for these diseases. Apart from its anti-malarial properties, artemisinin and its derivatives also exhibit potent anti-angiogenic properties. The molecular mechanisms underlying their inhibitory effects on angiogenesis have been studied by several groups. These investigations have revealed that artemisinins inhibit angiogenesis via the perturbations of cellular signaling pathways involved in the regulation of angiogenesis. Along with a brief introduction to artemisinin derivatives, this review provides a detailed summary of
\end{abstract}

Correspondence to: Professor Ju Liu, Laboratory of Microvascular Medicine, Medical Research Center, Shandong Provincial Qianfoshan Hospital, Shandong University, 16766 Jingshi Road, Jinan, Shandong 250014, P.R. China

E-mail: ju.liu@sdu.edu.cn

Abbreviations: 4E-BP1, eukaryotic translation initiation factor $4 \mathrm{E}$ binding protein; ANG, angiopoietin; AP-1, activator protein-1; ART, artesunate; Atf-2: activating transcription factor-2; COX-2, cyclooxygenase-2; DHA, dihydroartemisinin; EC, endothelial cell; eIF-4E, eukaryotic translation initiation factor 4E; ERK, extracellular signalregulated kinase; HIF, hypoxia inducible factor; HUVEC, human umbilical vein endothelial cell; IL-8, interleukin-8; IкB, inhibitor $\kappa \mathrm{B}$; JNK, c-Jun N-terminal kinase; MAPK, mitogen-activated protein kinase; MMP, matrix metalloprotease; mTOR, mammalian target of rapamycin; mTORC1, mammalian target of rapamycin complex 1; NF- $\kappa \mathrm{B}$, nuclear factor- $\kappa \mathrm{B}$; NO, nitric oxide; PDTC, pyrrolidine dithiocarbamate; PI3K, phosphatidylinositide 3-kinase; RA, rheumatoid arthritis; RAFLS, rheumatoid arthritis fibroblast-like synoviocytes; ROS, reactive oxygen species; TNF, tumor necrosis factor; VEGF, vascular endothelial growth factor; VEGFR2, vascular endothelial growth factor receptor 2

Key words: artemisinin, angiogenesis, mitogen-activated protein kinase pathway, Akt/phosphatidylinositide 3-kinase pathway, nuclear factor- $\kappa \mathrm{B}$ pathway the effects of artemisinins on the mitogen-activated protein kinase (MAPK) pathway, the nuclear factor- $\kappa \mathrm{B}(\mathrm{NF}-\kappa \mathrm{B})$ pathway and the phosphatidylinositide 3-kinase (PI3K)/protein kinase B (Akt)/mammalian target of rapamycin (mTOR) pathway. Due to the multiplicity of their actions on relevant signaling pathways, artemisinins are promising candidates with potential for use as anti-angiogenic agents for the treatment of related diseases or disorders.

\section{Contents}

1. Introduction

2. Derivatives of artemisinin and their characteristics

3. Mechanisms underlying the anti-angiogenic effects of artemisinin derivatives

4. Conclusion

\section{Introduction}

Angiogenesis refers to the process through which new blood vessels form from the pre-existing vasculature (1-8). It requires a complex interplay between angiogenic stimuli and angiogenic repressors, which leads to the controlled activation, proliferation, and migration of endothelial cells (ECs) (9-11). Normal angiogenesis plays a vital role in growth, development and wound healing $(9,10)$. However, angiogenesis is also initiated in a number of diseases due to the imbalanced production of angiogenic regulators (12). Pathological angiogenesis results in an aberrant vasculature which accelerates disease progression $(9,11)$. To elaborate, elevated levels of vascular endothelial growth factor (VEGF) are responsible for angiogenesis in cancer, a process that provides blood supply for tumor growth and metastasis (9,13-15). Likewise, the increased retinal expression of VEGF in response to hyperglycemic damage leads to ocular neovascularization which increases the risk of vision loss in diabetic retinopathy $(16,17)$. Similar observations also present in rheumatoid arthritis (RA) and macular degeneration $(9,14,18,19)$. In view of the pivotal role of angiogenesis in the pathogeneses of numerous diseases, the inhibition of angiogenesis by the use of anti-angiogenic agents has become an important therapeutic approach (20).

Artemisinin is extracted from the traditional Chinese medicine 'qinghao' (Artemisia Annua L.) $(9,18)$. Its derivatives are 
renowned for their potent anti-malarial effects and reliable safety records $(9,18)$. During the past decade, emerging evidence has indicated that artemisinins also serve as effective treatments for cancer (9,13-15). The effectiveness of artemisinins in cancer at least in part relies on the inhibition of tumor angiogenesis $(9,15)$. For example, the daily injection of dihydroartemisinin (DHA), a semi-synthetic derivative of artemisinin, reduces the density of the tumor vasculature and consequently impairs tumor growth in mouse models (21). Moreover, other derivatives, such as artesunate (ART) and artemether display similar anti-angiogenenic properties $(15,18,22)$. Hence, artemisinins demonstrate auspicious potential for use as novel treatments for a wider variety of angiogenesis related diseases (23). There is evidence to suggest that several signaling pathways, including the mitogen-activated protein kinase (MAPK) pathway, the nuclear factor $-\kappa \mathrm{B}(\mathrm{NF}-\kappa \mathrm{B})$ pathway, and the phosphatidylinositide 3-kinase (PI3K)/protein kinase $\mathrm{B}(\mathrm{Akt}) / \mathrm{mammalian}$ target of rapamycin (mTOR) pathway, may mediate the inhibitory effect of artemisinin derivatives on angiogenesis $(1,2,18,24,25)$. Beginning by describing the characteristics of artemisinin derivatives, this review provides a comprehensive explanation of the current literature regarding the molecular mechanisms underlying the effects of artemisinins on angiogenesis.

\section{Derivatives of artemisinin and their characteristics}

Following the isolation of artemisinin, the parent compound, semi-synthetic derivatives, such as artemether, arteetherand ART have been developed with improved pharmacokinetics (26-28). The lipid-based derivatives, artemether and arteether, are highly lipophilic $(15,29)$. While possessing longer half-lives than more hydrophilic artemisinins, both compounds are better at transpassing the blood-brain barrier $(15,29)$. Coupled with their anticancer activity, they may be exceptionally efficient in treating brain tumors $(15,29)$. Due to the addition of a hemisuccinate group, out of all the artemisinins, ART has the best water solubility and bioavailability (9). Experiments such as human umbilical vein endothelial cell (HUVEC) migration assays have confirmed that ART successfully inhibits angiogenesis induced by human melanoma cells with a much lower concentration $(3,30)$. Nevertheless, artemisone and artemiside, two relatively newer 10-alkylaminoartemisinin derivatives, seem to have superior efficacy compared to ART $(31,32)$. Although artemiside still has to undergo a toxicological evaluation, it has been well-established that artemisone has negligible toxicity, particularly neurotoxicity owing to its low lipophilicity (31-33).

Despite the variability in the structure and biological properties of artemisinin derivatives, all compounds are metabolized into DHA after being administered into the body (9). DHA can also be synthesized artificially and possesses additional water solubility; hence, it is the most studied artemisinin analog other than ART $(3,9)$. On the other hand, novel derivatives of artemisinins are constantly being developed with further refined pharmacological properties (34). As argued by Jung et al, non-acetal-based artemisinins have lower neurotoxicity than the above-mentioned acetal-based artemisinins (34). For example, dexoartemisinin- $\mathrm{C}_{60}$ conjugate, which comprises of a dexoartemisinin dimer and a fullerene cage demonstrates potent anti-angiogenic activity in chorioallantoic membrane assay with enhanced efficacy and expected lower toxicity (34).

\section{Mechanisms underlying the anti-angiogenic effects of artemisinin derivatives}

The MAPK pathway. MAPKs, encompassing extracellular signal-regulated kinases (ERKs), c-Jun N-terminal kinase (JNK) and p38 MAPK, are involved in a wide range of cellular activities $(1,35)$. ERKs, which regulate cell proliferation and survival, can be activated by downstream signals of VEGF (1). The binding of VEGF with its receptors on ECs stimulates a conformational change of the Ras protein, which subsequently leads to the phosphorylation of Raf (1). Activated Raf in turn phosphorylates MEK1/2, direct activators of ERKs (1). This cascade of signals eventually results in the promotion of EC proliferation and survival (1). Unlike ERK, JNK and p38 MAPK mediate both cytoprotective and cytotoxic processes (35-37). JNK is a pro-angiogenic protein which can be induced by cellular stressors, such as hypoxia or inflammation $(35,36)$. Upon activation, JNK phosphorylates the c-jun component of the activator protein-1 (AP-1), which results in the nuclear translocation of both c-jun and activating-transcription factor-2 (Atf-2) $(36,38)$. Consequently, the expression levels of pro-angiogenic stimuli, including VEGF, cyclooxygenase-2 (COX-2) and matrix metalloproteases (MMPs) are increased (20,36,38-41).p38 MAPK responds to stress-related extracellular stimuli in a similar manner (37). On the other hand, both JNK and p38 MAPK are key mediators of apoptosis $(11,42,43)$. It has been hypothesized that JNK is critical for the activation of pro-apoptotic protein Bax (43). In addition to Bax, p38 MAPK also upregulates the pro-apoptotic Fas, while inhibiting proteins that promote cell survival (ERK and Akt) (37).

The artemisinin family drugs act upon MAPK signaling cascades in multiple ways. DHA inhibits HUVEC proliferation by blocking both the transcription and activation of ERK1/2 (1). The incubation of HUVECs with DHA $(20 \mu \mathrm{M})$ for up to $12 \mathrm{~h}$ was shown to successfully reduce the expression of ERK1/2 at both the mRNA and protein level (1). Together with the decreased level of phosphorylated ERK1/2, these results were accompanied by a dose-dependent decrease in HUVEC proliferation (1) (Fig. 1). Moreover, the addition of PD98059, an inhibitor of MEK1/2, resulted in a comparable inhibition of HUVEC proliferation (1). Furthermore, the co-administration of DHA and PD98059 did not lead to further reduction in the proportion of proliferating HUVECs (1). Since ERK1/2 are activated by MEK1/2 only, the lack of additive effect between PD98059 and DHA justifies that DHA restrains angiogenesis by impeding ERK related cytoprotective activities (1) (Table I).

As previously demonstrated, DHA, at a concentration of $20 \mu \mathrm{M}$, significantly increased the level of activated JNK in HUVECs at $6 \mathrm{~h}$ of incubation (44). In addition, the level of activated $\mathrm{JNK}$ plateaued at $12 \mathrm{~h}$-incubation before starting to decline at $24 \mathrm{~h}$ (44). Intriguingly, although the activation of JNK is also involved in apoptosis, DHA exerted no effect on HUVEC viability (44). Nonetheless, beginning from a concentration of $12.5 \mu \mathrm{M}$, ART decreased the level of activated JNK in HUVECs following incubation for $0.5 \mathrm{~h}$ (11) (Fig. 1). Accordingly, the proliferation of ART-treated HUVECs was also inhibited (11). The results from these two studies rise controversy regarding the distinctions in effects of different artemisinin analogs on JNK activation (Table I). 
Table I. Mechanisms underlying the anti-angiogenesis effects of artemisinin derivatives.

In vitro experiments

\begin{tabular}{|c|c|c|c|c|}
\hline Analog & Cell type & Effect & Mechanism & (Refs.) \\
\hline \multicolumn{5}{|c|}{ Experiments on ECs } \\
\hline \multirow[t]{2}{*}{ ART } & \multirow[t]{2}{*}{ HUVECs } & Proliferation $\downarrow$ & JNK activation $\downarrow$ & $(11)$ \\
\hline & & Apoptosis $\uparrow$ & p38 MAPK activation $\uparrow$ & (11) \\
\hline \multirow[t]{4}{*}{ DHA } & \multirow[t]{4}{*}{ HUVECs } & Proliferation $\downarrow$ & ERK signalling $\downarrow$ & $(1)$ \\
\hline & & Migration $\downarrow$ & Independent of p38 MAPK activation & (3) \\
\hline & & Proliferation & VEGFR2 expression $\downarrow$ & (2) \\
\hline & & and migration $\downarrow$ & $\begin{array}{l}\text { Nuclear translocation and DNA } \\
\text { binding capacity of NF- } \mathrm{B} \downarrow\end{array}$ & $(2,21)$ \\
\hline \multicolumn{5}{|c|}{ Experiments on non-ECs } \\
\hline \multirow[t]{3}{*}{ ART } & \multirow[t]{3}{*}{ RAFLS } & \multirow{3}{*}{ IL-8 production $\downarrow$} & Akt phosphorylation $\downarrow$ & $(18)$ \\
\hline & & & $\begin{array}{l}\text { Nuclear translocation and DNA } \\
\text { binding capacity of NF- } \mathrm{B} \downarrow\end{array}$ & $(52)$ \\
\hline & & & Akt phosphorylation $\downarrow$ & $(52)$ \\
\hline DHA & $\begin{array}{l}\text { Rhabdomyosarcoma cells } \\
\text { Ewing sarcoma cells }\end{array}$ & VEGF production $\downarrow$ & Blockade of mTORC1 & $(24)$ \\
\hline
\end{tabular}

In vivo experiments

\begin{tabular}{|c|c|c|c|c|}
\hline Analog & Animal model & Effect & Mechanism & (Refs.) \\
\hline ART & Sprague-Dawley rats & Corneal neovascularization $\downarrow$ & p38 MAPK activation $\uparrow$ & (11) \\
\hline \multirow[t]{2}{*}{ DHA } & $\mathrm{BALB} / \mathrm{c}$ nude mice & $\begin{array}{l}\text { Production of pro-angiogenic cytokines } \downarrow \\
\text { Tumor microvessel density } \downarrow\end{array}$ & NF- $\kappa \mathrm{B}$ activity $\downarrow$ & $(21)$ \\
\hline & $\mathrm{C} 57 \mathrm{BL} / 6 \mathrm{~N}$ mice & Retinal neovascularization $\downarrow$ & $\mathrm{NF}-\kappa \mathrm{B}$ activity $\downarrow$ & (2) \\
\hline
\end{tabular}

EC, endothelial cell; ART, artesunate; HUVECs, human umbilical vein endothelial cells; JNK, c-jun N-terminal kinase; MAPK, mitogen activated protein kinase; DHA, dihydroartemisinin; ERK, extracellular signal-regulated kinase; VEGFR2, vascular endothelial growth factor receptor 2; $\mathrm{NF}-\kappa \mathrm{B}$, nuclear factor- $\mathrm{KB}$; RAFLS, rheumatoid arthritis fibroblast-like synoviocytes; VEGF, vascular endothelial growth factor; IL, interleukin; mTORC1, mammalian target of rapamycin complex 1. The upward arrows indicate the increase in the activities, and the downward arrows indicate the decrease in the activities.

In a previous study, compared to the phosphate-buffered saline (PBS)-treated controls, ART significantly increased the proportion of apoptotic HUVECs by inducing p38 MAPK activation (11). Further investigation revealed that activated p38 MAPK leads to an increase in the Bax/Bcl-2 ratio and the cleavage of caspase-9, which ultimately results in apoptosis via the intrinsic mitochondrial pathway (11) (Fig. 1). Moreover, pretreatment of HUVECs with a p38 MAPK inhibitor (SB203850) abolished the ART-induced activation of p38 MAPK, while it decreased the proportion of apoptotic cells (11) (Table I). Treatment with ART $(25 \mu \mathrm{M})$ was also able to reduce rat corneal neovascularization in response to alkaline burns (11). In addition, TUNEL and CD31 double staining of those corneal sections revealed a substantially larger proportion of apoptotic vascular ECs in the ART-treated group (11) (Table I). Therefore, both in vitro and in vivo experiments suggest that ART inhibits angiogenesis by activating p38 MAPK and promoting EC apoptosis (11). Notably, the pro-apoptotic effect of ART seems to rely on the formation of reactive oxygen species (ROS) via the cleavage of the endoperoxide bond by ferrous iron $(9,11,45,46)$. The reduced phosphorylation of p38 MAPK was observed simultaneously with the inhibition of ROS generation, whereas the addition of ferrous iron along with ART facilitated ROS production and increased the proportion of apoptotic ECs (11). Intriguingly, although the possession of an endoperoxide bond is a common feature of all aretemisinin derivatives, DHA $(20 \mu \mathrm{M})$ restricts EC proliferation and migration without inducing apoptosis $(1,3,15)$. Seeing the role of p38 MAPK in ART-induced EC apoptosis, such a result provides little evidence for DHA to have a comparable influence to ART on p38 MAPK signaling (11). Indeed, DHA $(20 \mu \mathrm{M})$ did not induce any change in the level of either p38 MAPK or activated p38 MAPK in HUVECs (3) (Fig. 1). Moreover, the blockade of p38 MAPK by SB203850 had no effect on the DHA-suppressed EC migration (3) (Table I). Therefore, unlike ART, DHA inhibits EC migration via a mechanism that is independent of p38 MAPK.

The NF- $\kappa B$ pathway. In addition to its role in innate immunity, $N F-\kappa B$ regulates the transcription of numerous angiogen- 


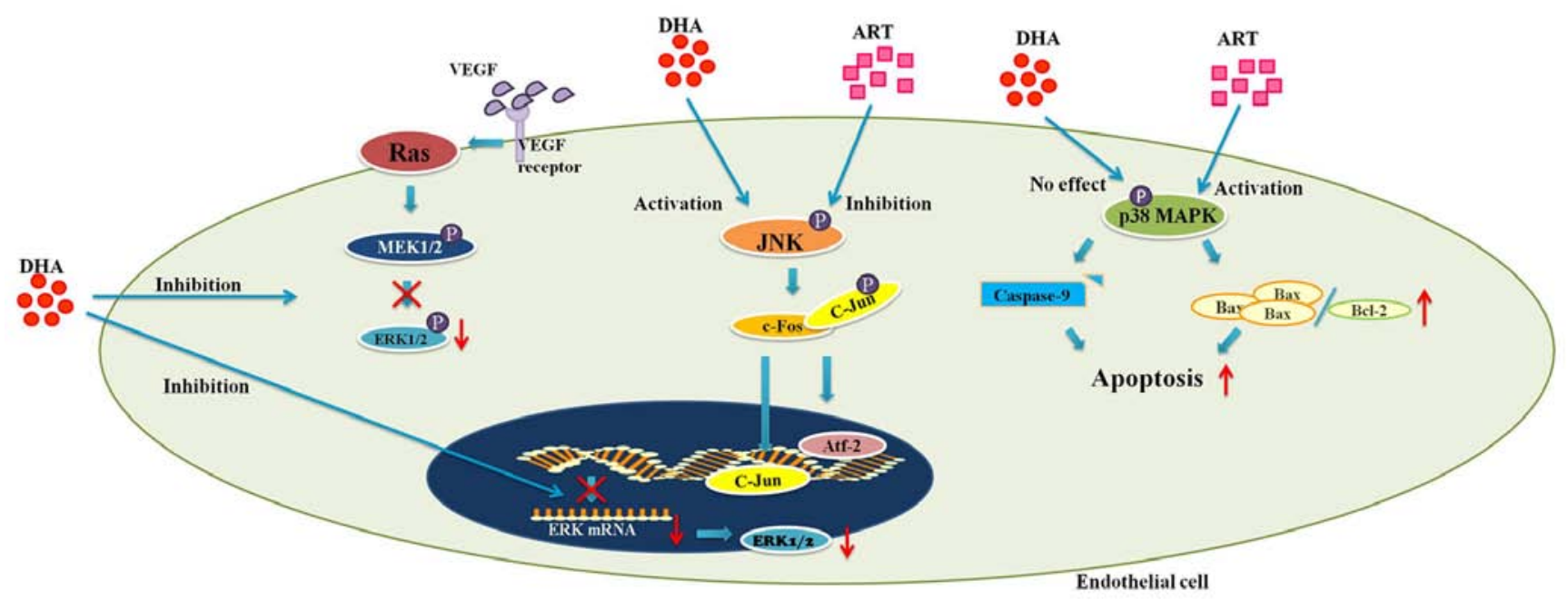

Figure 1. Effects of artemisinins on the mitogen-activated protein kinase (MAPK) cascades in endothelial cells.

esis-related genes (47). For instance, in hypoxia-induced angiogenesis, hypoxia inducible factor- $\alpha$ (HIF- $\alpha)$ becomes stabilized owing to the inhibition of prolyl hydroxylase-1, which leads to increased degradation of inhibitor $\kappa \mathrm{B}(\mathrm{I} \kappa \mathrm{B})(48)$. Since $\mathrm{I} \kappa \mathrm{B}$ is responsible for the masking of $\mathrm{NF}-\kappa \mathrm{B}(\mathrm{p} 65)$ nuclear localization sequence, its degradation results in the increased nuclear translocation of $\mathrm{NF}-\kappa \mathrm{B}$ and subsequently increased transcriptional expression of vascular endothelial growth factor receptor 2 (VEGFR2) $(2,48)$. VEGFR2 is crucial in mediating the VEGF-induced activation of pro-angiogenic signaling pathways (40). Hence, $\mathrm{NF}-\kappa \mathrm{B}$ plays an irreplaceable role in angiogenesis by regulating the production of VEGFR2 $(2,40)$.

On the other hand, the activation of $\mathrm{NF}-\kappa \mathrm{B}$ by other proinflammatory factors, such as tumor necrosis factor (TNF) may induce HIF-1 $\alpha$ under normoxic conditions (41). Indeed, many inflammation-related cytokines and chemokines modulated by $\mathrm{NF}-\kappa \mathrm{B}$ are pro-angiogenic factors (41). For example, in vitro experiments with HUVECs have shown that interleukin-8 (IL-8) promotes tube formation as well as EC infiltration (49). Similarly, the incubation of human appendix ECs with various chemokines has been shown to result in the formation of pseudovessels (50). Additionally, the activation of NF- $\kappa \mathrm{B}$ by lipopolysaccharides may directly upregulate HIF-2 and thus induce an increase in nitric oxide (NO) production (41). NO at a high concentration $(>1 \mu \mathrm{M})$ in turn stabilizes HIF- $\alpha$ expression and consequently increases the production of both VEGF and its receptors $(41,51)$.

There is extensive evidence to suggest that interactions between artemisinins and $\mathrm{NF}-\kappa \mathrm{B}$ signaling inhibit angiogenesis $(2,15,21)$. In particular, DHA was found to prevent the nuclear translocation of NF- $\kappa \mathrm{B}$ in HUVECs by increasing the I $\mathrm{B}$ level (2). Consequently, the production of VEGFR2 was decreased (2) (Fig. 2). Moreover, DHA downregulates the binding of NF- $\kappa \mathrm{B}$ p 65 to the promoter region of VEGFR2 (2). The lack of synergy between DHA and a known NF- $\kappa \mathrm{B}$ inhibitor [pyrrolidine dithiocarbamate (PDTC)] further confirms that DHA operates by interfering with the NF- $\kappa \mathrm{B}$ pathway (2). Considering the aforementioned role of VEGFR2, suppressed VEGFR2 production conceivably explains the reduced $\mathrm{EC}$ proliferation and migration following treatment with DHA (2). Moreover, the daily injection of DHA into the vitreous humor substantially reduced retinal neovascularization in mice models (2). Moreover, combined treatment with DHA and PDTC resulted in no further reduction in retinal vessel density (2) (Table I). Therefore, DHA inhibits angiogenesis in vitro and in vivo by blocking $\mathrm{NF}-\kappa \mathrm{B}$ signaling (2).

In the meantime, artemisinins exert their antiangiogenic effects by ameliorating NF- $\mathrm{B}-$ mediated inflammation $(9,21,52)$. Pretreating human RA fibroblastlike synoviocytes (RAFLS) with ART $(1 \mu \mathrm{M})$ significantly suppressed $\mathrm{NF}-\kappa \mathrm{B}$ mediated IL- 8 production induced by TNF- $\alpha$ (52). Following the addition of TNF- $\alpha$, ART prevented $\mathrm{I} \kappa \mathrm{B}$ degradation, leading to the reduced nuclear translocation and weakened DNA-binding capacity of NF- $\mathrm{BB}$ (52). In vitro experiments using HUVECs treated with DHA produced almost identical results (21) (Table I). Since IL-8 has long been recognized as a pro-angiogenic cytokine, it appears that artemisinins may inhibit angiogenesis by interfering with $\mathrm{NF}-\kappa \mathrm{B}$ signaling and consequently inhibiting IL-8 production (49). In addition, the daily injection of DHA into mice with xenografts of the pancreatic cancer cell line, BxPC-3, was shown to result in a dose-dependent reduction of VEGF, IL- 8 and COX-2 in tumor cells (21). Moreover, the reduced production of the above-mentioned pro-angiogenic cytokines was accompanied by reduced $\mathrm{NF}-\kappa \mathrm{B}$ activity and decreased tumor microvessel density (21) (Fig. 2) (Table I). Taken together, artemisinins inhibit angiogenesis by suppressing the secretion of $\mathrm{NF}-\kappa \mathrm{B}$ regulated pro-angiogenic cytokines $(21,49)$.

The PI3K/Akt/mTOR pathway. The role of PI3K and its downstream targets Akt/mTOR in angiogenesis involves the modulation of VEGF expression and other angiogenic stimuli such as NO and angiopoietins (ANGs) (53). In mammals, PI3K regulates the expression of mTOR which phosphorylates the eukaryotic translation initiation factor $4 \mathrm{E}$ binding protein (4E-BP1) (54). The phosphorylation of 4E-BP1 reduces the stability of a complex consisting of eukaryotic translation initiation factor 4E (eIF-4E) and 4E-BP1 (54). Since the eIF-4E/4E-BP1 complex inhibits HIF-1 $\alpha$ translation, phosphorylated mTOR leads to $4 \mathrm{E}-\mathrm{BP} 1$ activation which increases 


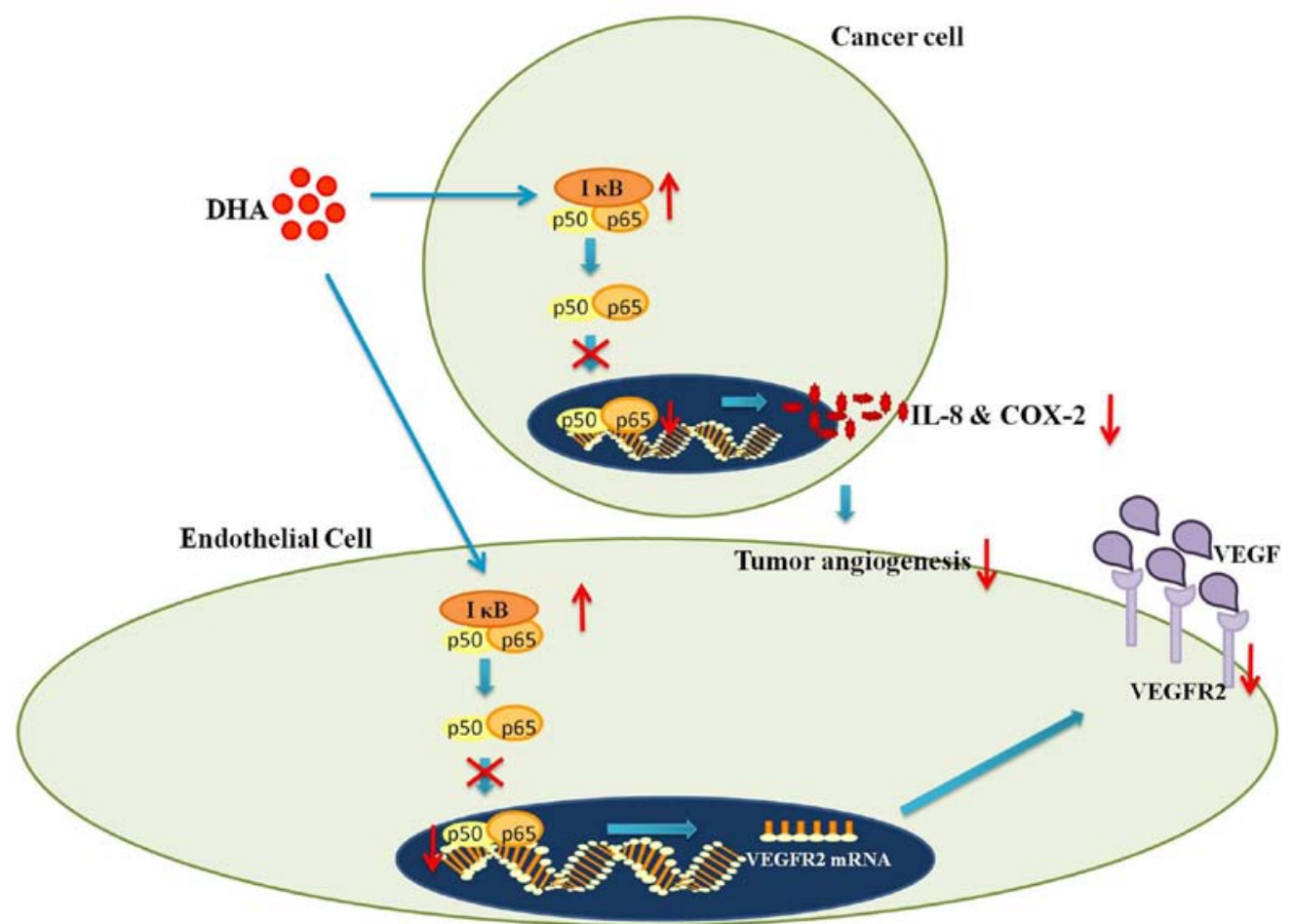

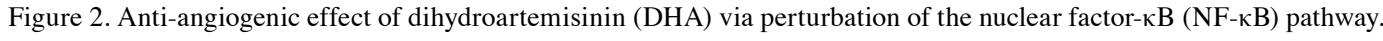

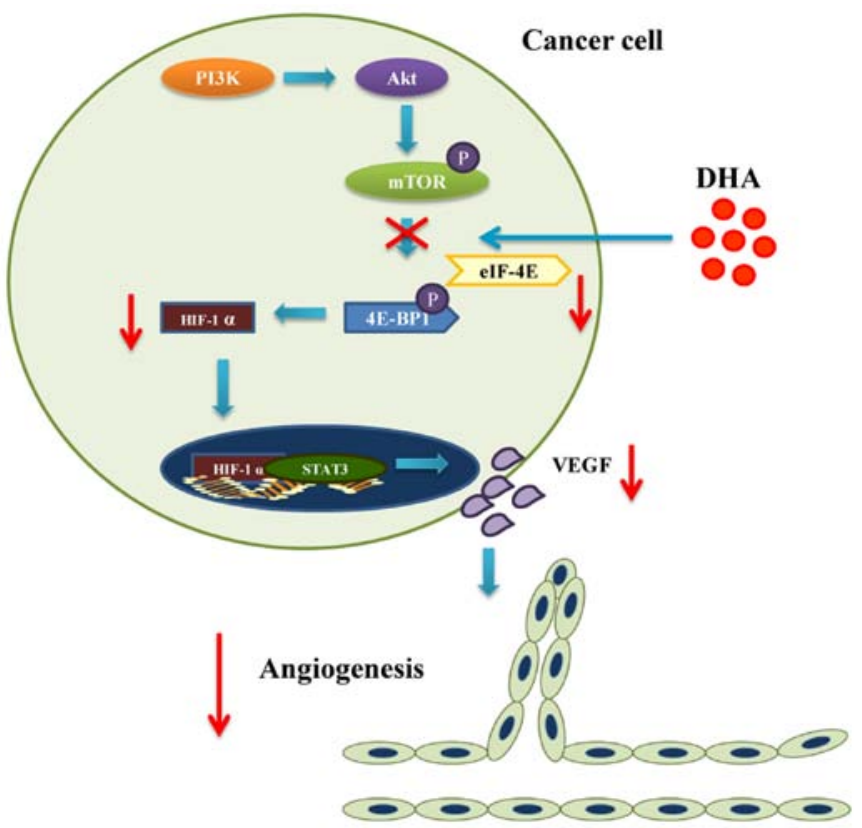

Figure 3. Predicted effect of dihydroartemisinin (DHA) on tumor angiogenesis mediated by the phosphatidylinositide 3-kinase (PI3K)/protein kinase B (Akt)/ mammalian target of rapamycin (mTOR) pathway.

the expression of HIF-1 $\alpha$ (54). In addition, Akt is able to activate endothelial NO synthase (eNOS), one of the regulators of NO synthesis in tumors (53). Activated eNOS mediates VEGF induced EC migration (53). Meanwhile, ANGs and their receptors are another class of growth factors facilitating the effect of VEGF that are related to the PI3K/Akt/mTOR pathway (53).

ART inhibits angiogenesis by preventing Akt activation. ART reduces the production of pro-inflammatory cytokines and VEGF in human RAFLS (18). To elaborate, PI3K inhibitor inhibits the production of several pro-inflammatory cytokines including the pro-angiogenic IL-8 (52). The inhibition of PI3K also correlates with reduced expression and nuclear translocation of HIF-1 $\alpha$ (18). Accordingly, the transcriptional expression of VEGF is decreased (18). There is evidence to suggest that ART prevents Akt phosphorylation, while hampering the production of VEGF and IL-8 in a similar manner $(18,52)$ (Table I). Apart from IL-8 and VEGF, the decreased phosphorylation of Akt is likely to diminish the effect of eNOS and ANG2 on angiogenesis. Therefore, inhibited Akt activation by ART leads to reduced production of angiogenic stimuli $(18,52)$.

Results from numerous studies have indicated that DHA also functions as a PI3K/Akt/mTOR inhibitor (55-57). Apart from inhibiting Akt activity, DHA primarily exerts its effect by interacting with mTOR $(24,58)$. DHA effectively blocks mTOR complex 1 (mTORC1) in rhabdomyosarcoma cells and Ewing sarcoma cells in both a dose- and time-dependent manner (24,25). As a result, binding between 4E-BP1 and eIF-4E is enhanced (24). Since tumor angiogenesis is powered by the sustained secretion of VEGF by tumor cells under hypoxic stress, it relies on the stabilization of HIF-1 $\alpha$ induced by degradation of 4E-BP1 eIF-4E complex (54). Blockade of mTORC1 by DHA impairs the ability of tumor cells to secrete VEGF, which arguably contributes to the inhibition of tumor angiogenesis (Fig. 3 and Table I).

The effects of artemisinins on selected signaling pathways may depend on cell types. For example, as previously demonstrated, ART activated none of the three MAPKs in TNF- $\alpha$ stimulated RAFLSs, which is in contrast with the findings using HUVECs $(11,18,52)$. Likewise, DHA inhibited ERK signaling in HUVECs but did not alter ERK signaling in cultured T cells (1,55). Overall, ECs seem to be especially susceptible to 
influences of artemisinins, which again signifies the potential for artemisinin derivatives to be used as anti-angiogenic agents. Moreover, the results mentioned above suggest that artemisinin derivatives may have distinct actions in different disease models. Hence, tailoring treatment schemes according to these variations may optimize the outcome.

\section{Conclusion}

Apart from anti-malaria, extensive evidence suggests that artemisinins inhibit angiogenesis. The effects of artemisinins on angiogenesis rely on perturbations of MAPK pathway, NF- $\kappa \mathrm{B}$ pathway, and PI3K/Akt/mTOR pathway $(1,2,11,21,52)$. DHA inhibits EC proliferation by reducing ERK1/2 expression and activation $(1,11)$. In the meantime, ART and DHA appear to play distinct roles in JNK and p38 MAPK activation $(3,11,44)$. In addition to decreasing VEGFR2 expression in ECs, artemisinins limit angiogenesis by mitigating the production of pro-angiogenic cytokines from tumor cells $(2,21,52)$. Both actions are achieved by the inhibition of $N F-\kappa B$ activity $(2,21,52)$. Furthermore, since artemisinins prevent activation of both Akt and mTOR, they are able to interfere with relevant downstream pro-angiogenic gene transcription to inhibit angiogenesis $(24,25,52,53,55-59)$. The pleiotropy of the effects of artemisinins renders them as potent anti-angiogenic agents. In view of the significance of angiogenesis in pathogeneses of many diseases, artemisinin and its derivatives are excellent candidates to be used in novel therapies (18).

\section{Acknowledgements}

This study was supported by grants from the National Natural Science Foundation of China (no. 81570255), and the Medical Science and Technology Development Plan of Shandong Province (no. 2013WS0137). We are grateful for the support from Shandong Taishan Scholarship (to Ju Liu).

\section{References}

1. Dong F, Tian H, Yan S, Li L, Dong X, Wang F, Li J, Li C, Cao Z, Liu X, et al: Dihydroartemisinin inhibits endothelial cell proliferation through the suppression of the ERK signaling pathway. Int J Mol Med 35: 1381-1387, 2015.

2. Dong F, Zhou X, Li C, Yan S, Deng X, Cao Z, Li L, Tang B, Allen TD and Liu J: Dihydroartemisinin targets VEGFR2 via the NF- $\mathrm{KB}$ pathway in endothelial cells to inhibit angiogenesis. Cancer Biol Ther 15: 1479-1488, 2014.

3. Guo L, Dong F, Hou Y, Cai W, Zhou X, Huang AL, Yang M, Allen TD and Liu J: Dihydroartemisinin inhibits vascular endothelial growth factor-induced endothelial cell migration by a p38 mitogen-activated protein kinase-independent pathway. Exp Ther Med 8: 1707-1712, 2014.

4. Oh S, Jeong IH, Shin WS and Lee S: Growth inhibition activity of thioacetal artemisinin derivatives against human umbilical vein endothelial cells. Bioorg Med Chem Lett 13: 3665-3668, 2003.

5. Oh S, Jeong IH, Ahn CM, Shin WS and Lee S: Synthesis and antiangiogenic activity of thioacetal artemisinin derivatives. Bioorg Med Chem 12: 3783-3790, 2004.

6. Oh S, Jeong IH, Shin WS and Lee S: Synthesis and antiangiogenic activity of exo-olefinated deoxoartemisinin derivatives. Bioorg Med Chem Lett 14: 3683-3686, 2004.

7. Ricci J, Park J, Chung WY, Park KK and Jung M: Concise synthesis and antiangiogenic activity of artemisinin-glycolipid hybrids on chorioallantoic membranes. Bioorg Med Chem Lett 20: 6858-6860, 2010.

8. Risau W: Mechanisms of angiogenesis. Nature 386: 671-674, 1997.
9. Ho WE, Peh HY, Chan TK and Wong WS: Artemisinins: Pharmacological actions beyond anti-malarial. Pharmacol Ther 142: 126-139, 2014.

10. Carmeliet P and Jain RK: Molecular mechanisms and clinical applications of angiogenesis. Nature 473: 298-307, 2011.

11. Cheng R, Li C, Li C, Wei L, Li L, Zhang Y, Yao Y, Gu X, Cai W, Yang Z, et al: The artemisinin derivative artesunate inhibits corneal neovascularization by inducing ROS-dependent apoptosis in vascular endothelial cells. Invest Ophthalmol Vis Sci 54: 3400-3409, 2013.

12. Nagy JA, Dvorak AM and Dvorak HF: VEGF-A and the induction of pathological angiogenesis. Annu Rev Pathol 2: 251-275, 2007.

13. Ferrara N: VEGF and the quest for tumour angiogenesis factors. Nat Rev Cancer 2: 795-803, 2002.

14. Ferrara N: VEGF-A: A critical regulator of blood vessel growth. Eur Cytokine Netw 20: 158-163, 2009.

15. Crespo-Ortiz MP and Wei MQ: Antitumor activity of artemisinin and its derivatives: From a well-known antimalarial agent to a potential anticancer drug. J Biomed Biotechnol 2012: 247597, 2012.

16. Arden GB, Wolf JE and Tsang Y: Does dark adaptation exacerbate diabetic retinopathy? Evidence and a linking hypothesis. Vision Res 38: 1723-1729, 1998.

17. Crawford TN, Alfaro DV III, Kerrison JB and Jablon EP: Diabetic retinopathy and angiogenesis. Curr Diabetes Rev 5: 8-13, 2009.

18. He Y, Fan J, Lin H, Yang X, Ye Y, Liang L, Zhan Z, Dong X, Sun $\mathrm{L}$ and $\mathrm{Xu} \mathrm{H}$ : The anti-malaria agent artesunate inhibits expression of vascular endothelial growth factor and hypoxiainducible factor- $1 \alpha$ in human rheumatoid arthritis fibroblast-like synoviocyte. Rheumatol Int 31: 53-60, 2011.

19. Folkman J: Angiogenesis in cancer, vascular, rheumatoid and other disease. Nat Med 1: 27-31, 1995.

20. Polverini PJ: Angiogenesis in health and disease: Insights into basic mechanisms and therapeutic opportunities. J Dent Educ 66: 962-975, 2002.

21. Wang SJ, Sun B, Cheng ZX, Zhou HX, Gao Y, Kong R, Chen H, Jiang HC, Pan SH, Xue DB, et al: Dihydroartemisinin inhibits angiogenesis in pancreatic cancer by targeting the NF- $\kappa \mathrm{B}$ pathway. Cancer Chemother Pharmacol 68: 1421-1430, 2011.

22. Jeong E, Song HJ, Lim S, Lee SJ, Lim JE, Nam DH, Joo KM, Jeong BC, Jeon SS, Choi HY, et al: Repurposing the anti-malarial drug artesunate as a novel therapeutic agent for metastatic renal cell carcinoma due to its attenuation of tumor growth, metastasis, and angiogenesis. Oncotarget 6: 33046-33064, 2015.

23. Zhu XX, Yang L, Li YJ, Zhang D, Chen Y, Kostecká P, Kmoníčková E and Zídek Z: Effects of sesquiterpene, flavonoid and coumarin types of compounds from Artemisia annua $\mathrm{L}$. on production of mediators of angiogenesis. Pharmacol Rep 65: 410-420, 2013.

24. Odaka Y, Xu B, Luo Y, Shen T, Shang C, Wu Y, Zhou H and Huang S: Dihydroartemisinin inhibits the mammalian target of rapamycin-mediated signaling pathways in tumor cells. Carcinogenesis 35: 192-200, 2014.

25. Hay N and Sonenberg N: Upstream and downstream of mTOR. Genes Dev 18: 1926-1945, 2004.

26. Corsello MA and Garg NK: Synthetic chemistry fuels interdisciplinary approaches to the production of artemisinin. Nat Prod Rep 32: 359-366, 2015.

27. Mott BT, He R, Chen X, Fox JM, Civin CI, Arav-Boger R and Posner GH: Artemisinin-derived dimer phosphate esters as potent anti-cytomegalovirus (anti-CMV) and anticancer agents: A structure-activity study. Bioorg Med Chem 21: 3702-3707, 2013.

28. Lee S: Artemisinin, promising lead natural product for various drug developments. Mini Rev Med Chem 7: 411-422, 2007.

29. Singh NP and Panwar VK: Case report of a pituitary macroadenoma treated with artemether. Integr Cancer Ther 5: 391-394, 2006.

30. Chen H, Shi L, Yang X, Li S, Guo X and Pan L: Artesunate inhibiting angiogenesis induced by human myeloma RPMI-8226 cells. Int J Hematol 92: 587-597, 2010.

31. Nagelschmitz J, Voith B, Wensing G, Roemer A, Fugmann B, Haynes RK, Kotecka BM, Rieckmann KH and Edstein MD: First assessment in humans of the safety, tolerability, pharmacokinetics, and ex vivo pharmacodynamic antimalarial activity of the new artemisinin derivative artemisone. Antimicrob Agents Chemother 52: 3085-3091, 2008.

32. Ansari MT, Saify ZS, Sultana N, Ahmad I, Saeed-Ul-Hassan S, Tariq I and Khanum M: Malaria and artemisinin derivatives: An updated review. Mini Rev Med Chem 13: 1879-1902, 2013. 
33. Haynes RK, Fugmann B, Stetter J, Rieckmann K, Heilmann HD, Chan HW, Cheung MK, Lam WL, Wong HN, Croft SL, et al: Artemisone - a highly active antimalarial drug of the artemisinin class. Angew Chem Int Ed Engl 45: 2082-2088, 2006.

34. Jung M, Tak J, Chung WY and Park KK: Antiangiogenic activity of deoxoartemisinin derivatives on chorioallantoic membrane. Bioorg Med Chem Lett 16: 1227-1230, 2006.

35. Shen K, Ji L, Lu B and Wang Z: c-Jun N-terminal kinase mediated VEGFR2 sustained phosphorylation is critical for VEGFA-induced angiogenesis in vitro and in vivo. Cell Biochem Biophys 64: 17-27, 2012.

36. Miura S, Matsuo Y and Saku K: Jun N-terminal kinase inhibitor blocks angiogenesis by blocking VEGF secretion and an MMP pathway. J Atheroscler Thromb 15: 69-74, 2008.

37. Grossi V, Peserico A, Tezil T and Simone C: p38a MAPK pathway: A key factor in colorectal cancer therapy and chemoresistance. World J Gastroenterol 20: 9744-9758, 2014.

38. Li Z, Meng D, Li G, Xu J, Tian K and Li Y: Celecoxib combined with diacerein effectively alleviates osteoarthritis in rats via regulating JNK and p38MAPK signaling pathways. Inflammation 38: $1563-1572,2015$

39. Ma X, Liu Y, Wang Q, Chen Y, Liu M, Li X, Xiang R, Wei Y, Duan Y and Han J: Tamoxifen induces the development of hernia in mice by activating MMP-2 and MMP-13 expression. Biochim Biophys Acta 1852: 1038-1048, 2015.

40. Sato Y, Kanno S, Oda N, Abe M, Ito M, Shitara K and Shibuya M Properties of two VEGF receptors, Flt-1 and KDR, in signal transduction. Ann NY Acad Sci 902: 201-207, 2000.

41. Szade A, Grochot-Przeczek A, Florczyk U, Jozkowicz A and Dulak J: Cellular and molecular mechanisms of inflammationinduced angiogenesis. IUBMB Life 67: 145-159, 2015.

42. Gupta K, Kshirsagar S, Li W, Gui L, Ramakrishnan S, Gupta P, Law PY and Hebbel RP: VEGF prevents apoptosis of human microvascular endothelial cells via opposing effects on MAPK/ERK and SAPK/JNK signaling. Exp Cell Res 247: 495-504, 1999.

43. Weston CR and Davis RJ: The JNK signal transduction pathway. Curr Opin Cell Biol 19: 142-149, 2007.

44. Dong F, Han J, Jing G, Chen X, Yan S, Yue L, Cao Z, Liu X, Ma G and Liu J: Dihydroartemisinin transiently activates the JNK/SAPK signaling pathway in endothelial cells. Oncol Lett 12: 4699-4704, 2016.

45. Firestone GL and Sundar S: Anticancer activities of artemisinin and its bioactive derivatives. Expert Rev Mol Med 11: e32, 2009.

46. Devasagayam TP, Tilak JC, Boloor KK, Sane KS, Ghaskadbi SS and Lele RD: Free radicals and antioxidants in human health: Current status and future prospects. J Assoc Physicians India 52: 794-804, 2004

47. Hayden MS and Ghosh S: Shared principles in NF-kappaB signaling. Cell 132: 344-362, 2008.
48. Oliver KM, Taylor CT and Cummins EP: Hypoxia. Regulation of NFkappaB signalling during inflammation: The role of hydroxylases. Arthritis Res Ther 11: 215, 2009.

49. Hsiao KY, Chang N, Lin SC, Li YH and Wu MH: Inhibition of dual specificity phosphatase-2 by hypoxia promotes interleukin8-mediated angiogenesis in endometriosis. Hum Reprod 29: 2747-2755, 2014

50. Suffee N, Richard B, Hlawaty H, Oudar O, Charnaux N and Sutton A: Angiogenic properties of the chemokine RANTES/CCL5. Biochem Soc Trans 39: 1649-1653, 2011.

51. Mateo J, García-Lecea M, Cadenas S, Hernández C and Moncada S: Regulation of hypoxia-inducible factor-1alpha by nitric oxide through mitochondria-dependent and -independent pathways. Biochem J 376: 537-544, 2003.

52. Xu H, He Y, Yang X, Liang L, Zhan Z, Ye Y, Yang X, Lian F and Sun L: Anti-malarial agent artesunate inhibits TNF-alphainduced production of proinflammatory cytokines via inhibition of NF-kappaB and PI3 kinase/Akt signal pathway in human rheumatoid arthritis fibroblast-like synoviocytes. Rheumatology (Oxford) 46: 920-926, 2007.

53. Karar J and Maity A: PI3K/AKT/mTOR pathway in angiogenesis. Front Mol Neurosci 4: 51, 2011.

54. Masoud GN and Li W: HIF-1 $\alpha$ pathway: Role, regulation and intervention for cancer therapy. Acta Pharm Sin B 5: 378-389, 2015.

55. Zhao YG, Wang Y, Guo Z, Gu AD, Dan HC, Baldwin AS, Hao W and Wan YY: Dihydroartemisinin ameliorates inflammatory disease by its reciprocal effects on Th and regulatory $\mathrm{T}$ cell function via modulating the mammalian target of rapamycin pathway. J Immunol 189: 4417-4425, 2012.

56. Chen Q, Chen L, Wu X, Zhang F, Jin H, Lu C, Shao J, Kong D, Wu L and Zheng S: Dihydroartemisinin prevents liver fibrosis in bile duct ligated rats by inducing hepatic stellate cell apoptosis through modulating the PI3K/Akt pathway. IUBMB Life 68: 220-231, 2016.

57. Feng $\mathrm{X}$, Li L, Jiang $\mathrm{H}$, Jiang $\mathrm{K}$, Jin $\mathrm{Y}$ and Zheng $\mathrm{J}$ : Dihydroartemisinin potentiates the anticancer effect of cisplatin via mTOR inhibition in cisplatin-resistant ovarian cancer cells: Involvement of apoptosis and autophagy. Biochem Biophys Res Commun 444: 376-381, 2014

58. Liao K, Li J and Wang Z: Dihydroartemisinin inhibits cell proliferation via AKT/GSK3 $3 /$ cyclinD1 pathway and induces apoptosis in A549 lung cancer cells. Int J Clin Exp Pathol 7: 8684-8691, 2014

59. Tan SS, Ong B, Cheng C, Ho WE, Tam JK, Stewart AG, Harris T, Wong WS and Tran T: The antimalarial drug artesunate inhibits primary human cultured airway smooth muscle cell proliferation. Am J Respir Cell Mol Biol 50: 451-458, 2014. 